\title{
General dynamical density functional theory for classical fluids
}

\author{
Benjamin D. Goddard, ${ }^{1}$ Andreas Nold, ${ }^{1,2}$ Nikos Savva, ${ }^{1}$ Grigorios A. Pavliotis,,${ }^{3,4}$ and Serafim Kalliadasis ${ }^{1}$ \\ ${ }^{1}$ Department of Chemical Engineering, Imperial College London, London, SW7 2AZ, United Kingdom \\ ${ }^{2}$ Center of Smart Interfaces, TU Darmstadt, Petersenstr. 32, 64287 Darmstadt, Germany \\ ${ }^{3}$ Department of Mathematics, Imperial College London, London, SW7 2AZ, United Kingdom \\ ${ }^{4}$ Institut für Mathematik, Freie Universität Berlin, Arnimallee 6, 14195 Berlin, Germany
}

(Dated: November 15, 2018)

\begin{abstract}
We study the dynamics of a colloidal fluid including inertia and hydrodynamic interactions, two effects which strongly influence the non-equilibrium properties of the system. We derive a general dynamical density functional theory (DDFT) which shows very good agreement with full Langevin dynamics. In suitable limits, we recover existing DDFTs and a Navier-Stokes-like equation with additional non-local terms.
\end{abstract}

PACS numbers: 47.57.J-, 83.10.Mj, 83.80.Hj, 61.20.Lc

Since the observation of the Brownian motion of pollen particles in water in the 19th Century [1, the study of classical fluids has been fundamental not only to the development of statistical mechanics [2, but also to many other fields in physics, chemistry and engineering, e.g. the evolution of microscopy over the last century [3], recent advances in biophysical research 4 and the rapidlygrowing field of microfluidics [5].

Colloidal systems, in particular, are versatile model ones for both theoretical and experimental scrutiny. Many of the forces governing their structure and behaviour govern also those of matter, whilst the sufficiently large physical size of colloidal particles makes them accessible experimentally. However, the large number of particles in real-world systems translates to highdimensional mathematical models, which quickly become computationally intractable.

Non-equilibrium statistical mechanics approaches 6 7], such as the Boltzmann equation, allow the dynamics of systems of arbitrarily large numbers of particles to be studied. An important example is dynamical density functional theory (DDFT) [7 for the evolution of the one-body mass distribution. However, existing DDFTs neglect either the momentum of the colloidal particles 8$]$, or the hydrodynamic interactions (HI) mediated through the bath 9, or both, as in the pioneering work in 10. Yet, inertial effects are negligible only in the high-friction limit [11, whilst HI are long range [12; it is therefore unclear that existing DDFTs are sufficiently accurate to model general colloidal systems. Here we outline a DDFT formalism which carefully and systematically accounts for inertia and HI, an important step towards accurate and predictive modelling of physically-relevant systems. It is validated with stochastic simulations, and existing DDFTs [8 10] are shown to be special cases.

We are interested in systems with a large number $N$ identical, spherically symmetric colloidal particles of mass $m$ suspended in a bath of many more, much smaller and much lighter particles. Typically, colloidal particles are of size $1 \mathrm{~nm}-1 \mu \mathrm{m}$, occupying the same volume as ap- proximately $10^{7}-10^{10}$ water molecules. As such, treating the bath particles exactly is computationally prohibitive. However, a typical timescale for a colloidal particle to diffuse a distance equal to its diameter is $1 \mathrm{~s}$, whilst the typical time between collisions of water molecules is $\tau_{b} \approx 10^{-15} \mathrm{~s} \quad[13$. Hence, for timescales significantly larger than $\tau_{b}$, we may introduce a coarsegrained model and consider only the colloidal particles, treating the bath in a stochastic manner.

This approximation leads to the Langevin [2] equations for the $3 N$-dimensional colloidal position and momentum vectors $\mathbf{r}=\left(\mathbf{r}_{1}, \ldots, \mathbf{r}_{N}\right)$ and $\mathbf{p}=\left(\mathbf{p}_{1}, \ldots, \mathbf{p}_{N}\right)$ with $\mathbf{r}_{i}$ and $\mathbf{p}_{i}$ the position and momentum of the $i$ th particle:

$$
\frac{\mathrm{d} \mathbf{r}}{\mathrm{d} t}=\frac{\mathbf{p}}{m}, \quad \frac{\mathrm{d} \mathbf{p}}{\mathrm{d} t}=-\nabla_{\mathbf{r}} V(\mathbf{r}, t)-\mathbf{\Gamma}(\mathbf{r}) \mathbf{p}+\mathbf{A}(\mathbf{r}) \mathbf{f}(t) .
$$

Here, $V$ is the potential, generally a sum of an external potential, such as gravity, and inter-particle potentials, such as electrostatic effects. The motion of the colloidal particles causes flows in the bath, which in turn cause forces on the colloidal particles, referred to already as HI. The momenta and forces are related by the $3 N \times 3 N$ positive-definite friction tensor $\boldsymbol{\Gamma}$. See Supplemental Material for demonstrations of these effects on sedimenting hard spheres. Finally, collisions of bath particles with colloidal particles are described by stochastic forces $\mathbf{f}$, given by Gaussian white noise, the strength of which is determined by a generalized fluctuation-dissipation theorem 14, $\mathbf{A}(\mathbf{r})=\left(m k_{B} T \boldsymbol{\Gamma}(\mathbf{r})\right)^{1 / 2}$, with $T$ the temperature and $k_{B}$ Boltzmann's constant. We assume that $T$ is constant in space, i.e. that the solvent bath is also a heat bath on colloidal timescales.

When $N$ is large, interest lies not in particular realizations of (1), or experiments, but in averages over a large number of them. Averaging (1) over the initial particle distribution and the noise leads to the Kramers (FokkerPlanck) equation, a $6 N$-dimensional deterministic PDE for the evolution of the distribution function $f^{(N)}(\mathbf{r}, \mathbf{p}, t)$, the probability of finding the particles with positions $\mathbf{r}$ 
and momenta $\mathbf{p}$ at time $t$ :

$$
\begin{aligned}
& {\left[\partial_{t}+\frac{1}{m} \mathbf{p} \cdot \nabla_{\mathbf{r}}-\nabla_{\mathbf{r}} V(\mathbf{r}, t) \cdot \boldsymbol{\nabla}_{\mathbf{p}}\right] f^{(N)}(\mathbf{r}, \mathbf{p}, t)} \\
& \quad-\boldsymbol{\nabla}_{\mathbf{p}} \cdot\left[\boldsymbol{\Gamma}(\mathbf{r})\left(\mathbf{p}+m k_{B} T \boldsymbol{\nabla}_{\mathbf{p}}\right) f^{(N)}(\mathbf{r}, \mathbf{p}, t)\right]=0
\end{aligned}
$$

The main issue with solving (1) or (2), as with any molecular approach, is that of computational intensity for large systems. For (2), taking $M$ discretization points for each dimension would require $M^{6 N}$ points. Hence, the only way to solve (2) for many particles is via Monte Carlo methods, i.e. by solving (1). However, for non-trivial HI this requires $\mathcal{O}\left(N^{3}\right)$ operations at each timestep, prohibiting calculations for many-particle systems. (Additionally, the characteristic scale of the spatial structures is often too large to be accurately treated.)

In contrast, it is known rigorously [15] that the $N$-body distribution function $f^{(N)}$ is a functional of the one-body position distribution $\rho\left(\mathbf{r}_{1}, t\right)=N \int \mathrm{d} \mathbf{p} \mathrm{d} \mathbf{r}^{\prime} f^{(N)}(\mathbf{r}, \mathbf{p}, t)$, where $\mathrm{d} \mathbf{r}^{\prime}$ denotes integration over all positions except $\mathbf{r}_{1}$. Hence, for any number of particles, the system is, in principle, completely described by a function of only a single three-dimensional position variable (cf. TDDFT in quantum mechanics [16]).

This motivates the derivation of a DDFT, a closed evolution equation for $\rho$. We consider the moments of (2) with respect to momentum and obtain an infinite hierarchy of equations, which must be truncated. This is analogous to deriving the Euler or Navier-Stokes equation from the Boltzmann equation [17. We truncate the hierarchy at the second equation; the next level treats the local temperature, which here is constant due to the heat bath. However, if required, this method can be systematically extended to higher levels of the hierarchy.

We obtain a continuity equation for the density

$$
\partial_{t} \rho\left(\mathbf{r}_{1}, t\right)+\nabla_{\mathbf{r}_{1}} \cdot\left(\rho\left(\mathbf{r}_{1}, t\right) \mathbf{v}\left(\mathbf{r}_{1}, t\right)\right)=0
$$

and an evolution equation for the local velocity $\mathbf{v}(\mathbf{r}, t)=$ $m^{-1} \int \mathrm{d} \mathbf{p} \mathrm{d} \mathbf{r}^{\prime} \mathbf{p} f^{(N)}(\mathbf{r}, \mathbf{p}, t)$ :

$$
\begin{aligned}
& D_{t} \mathbf{v}\left(\mathbf{r}_{1}, t\right)+\frac{1}{\rho\left(\mathbf{r}_{1}, t\right)} \boldsymbol{\nabla}_{\mathbf{r}_{1}} \cdot \int \mathrm{d} \mathbf{p}_{1} \frac{\mathbf{p}_{1} \otimes \mathbf{p}_{1}}{m^{2}} f_{\text {neq }}^{(1)}\left(\mathbf{r}_{1}, \mathbf{p}_{1}, t\right) \\
= & -\frac{1}{m} \boldsymbol{\nabla}_{\mathbf{r}_{1}} \frac{\delta \mathcal{F}[\rho]}{\delta \rho}-\gamma \mathbf{v}\left(\mathbf{r}_{1}, t\right) \\
& -\gamma \int \mathrm{d} \mathbf{r}_{2} \rho\left(\mathbf{r}_{2}, t\right) g\left(\mathbf{r}_{1}, \mathbf{r}_{2},[\rho]\right) \sum_{j=1}^{2} \mathbf{Z}_{j}\left(\mathbf{r}_{1}, \mathbf{r}_{2}\right) \mathbf{v}\left(\mathbf{r}_{j}, t\right)
\end{aligned}
$$

Here $D_{t}=\partial_{t}+\mathbf{v}\left(\mathbf{r}_{1}, t\right) \cdot \nabla_{\mathbf{r}_{1}}$ is the material derivative and $\mathcal{F}$ is the (equilibrium) Helmholtz free energy functional; see later. For ease of exposition, we restrict to two-body HI: $\boldsymbol{\Gamma}(\mathbf{r})=\gamma[\mathbf{1}+\tilde{\boldsymbol{\Gamma}}(\mathbf{r})]$, with the HI tensor $\tilde{\boldsymbol{\Gamma}}$ decomposed into $3 \times 3$ blocks $\tilde{\boldsymbol{\Gamma}}_{i j}(\mathbf{r})=$ $\delta_{i j} \sum_{\ell \neq i} \mathbf{Z}_{1}\left(\mathbf{r}_{i}, \mathbf{r}_{\ell}\right)+\left(1-\delta_{i j}\right) \mathbf{Z}_{2}\left(\mathbf{r}_{i}, \mathbf{r}_{j}\right)$ [11. Here $\mathbf{1}$ is the $3 N \times 3 N$ identity matrix and $\gamma$ is the friction felt by a single, isolated particle. Physically, $\tilde{\boldsymbol{\Gamma}}_{i j}$ describes the force on particle $i$ due to the momentum of particle $j$. This two-body formulation is generally more accurate than that for the diffusion tensor (as in [8]), which can lead to incorrect physics [18. We have decomposed the one-body distribution $f^{(1)}\left(\mathbf{r}_{1}, \mathbf{p}_{1}, t\right)=N \int \mathrm{d} \mathbf{p}^{\prime} \mathrm{d} \mathbf{r}^{\prime} f^{(N)}(\mathbf{r}, \mathbf{p}, t)=$ $f_{\text {le }}^{(1)}\left(\mathbf{r}_{1}, \mathbf{p}_{1}, t\right)+f_{\text {neq }}^{(1)}\left(\mathbf{r}_{1}, \mathbf{p}_{1}, t\right)$, where $f_{\text {le }}^{(1)}$ is the localequilibrium part, the momentum dependence of which is given by a local Maxwellian with mass $\rho(\mathbf{r}, t)$, mean $m \rho(\mathbf{r}, t) \mathbf{v}(\mathbf{r}, t)$ and variance $m k_{B} T \rho(\mathbf{r}, t)$. The corresponding three quantities are zero for the nonequilibrium part $f_{\text {neq. }}^{(1)}$. We have also written the twobody reduced distribution as $f^{(2)}\left(\mathbf{r}_{1}, \mathbf{r}_{2}, \mathbf{p}_{1}, \mathbf{p}_{2}, t\right)=$ $f^{(1)}\left(\mathbf{r}_{1}, \mathbf{p}_{1}, t\right) f^{(1)}\left(\mathbf{r}_{1}, \mathbf{p}_{1}, t\right) g\left(\mathbf{r}_{1}, \mathbf{r}_{2},[\rho]\right)[6]$.

The non-local terms in (4), absent from previous DDFTs, model important physical effects. That involving $\mathbf{Z}_{1}$ combines with $\gamma \mathbf{v}$ to give an effective, densitydependent friction coefficient. The $\mathbf{Z}_{2}$ term non-locally couples the velocities. Surprisingly, this does not require explicit momentum correlations in $g$. Neglecting these terms and setting $f_{\text {neq }}^{(1)}=0$ recovers a previous DDFT [9]. Setting $\gamma=0$ gives a DDFT for atomic and molecular fluids, although the closures below are harder to justify.

The non-trivial challenge here is to close the momentum equation (see Supplemental Material) as a functional of $\rho$. We briefly describe three steps:

At equilibrium there exists an exact functional identity [19] $N \int \mathrm{d} \mathbf{r}^{\prime} \nabla_{\mathbf{r}_{1}} V(\mathbf{r}) \rho^{(N)}(\mathbf{r})=$ $\left(\nabla_{\mathbf{r}_{1}} \frac{\delta \mathcal{F}[\rho]}{\delta \rho}-k_{B} T \nabla_{\mathbf{r}_{1}}\right) \rho\left(\mathbf{r}_{1}\right)$, where $\mathcal{F}[\rho]=$ $k_{B} T \int \mathrm{d} \mathbf{r}_{1} \rho\left(\mathbf{r}_{1}\right)\left[\ln \left(\Lambda^{3} \rho\left(\mathbf{r}_{1}\right)\right)-1\right] \quad+\quad \mathcal{F}_{\text {exc }}[\rho]+$ $\int \mathrm{d} \mathbf{r}_{1} \rho\left(\mathbf{r}_{1}\right) V_{1}\left(\mathbf{r}_{1}\right)$ with $\Lambda$ the (irrelevant) de Broglie wavelength. In general, $\mathcal{F}_{\text {exc }}$ (the excess over ideal gas term) is unknown but has been well-studied at equilibrium and good approximations exist, e.g. fundamental measure theory [7, 20] (accurate for hard spheres) and mean field theory 7], (exact for soft interactions at high densities). We thus assume that the same identity holds out of equilibrium, in particular giving the correct equilibrium behaviour.

Since HI vanish at equilibrium there exists no analogous identity. Instead, we assume the form for $f^{(2)}$ given above for a known functional $\mathrm{g}$. To go beyond this twobody approach it is necessary to obtain higher-order reduced distributions as functionals of $\rho$.

The term in (4) containing $f_{\text {neq }}^{(1)}$ is analogous to the kinetic pressure tensor [ [6], and there is no reason to expect it to be a simple functional of $\rho$ and $\mathbf{v}$. However, if it may be neglected (e.g. via a maximum entropy approach [21]) or approximated as a functional of $\rho$ and $\mathbf{v}$ (e.g. via a Chapman-Enskog expansion), (3) and (4) give a DDFT. Alternatively, extending the above hierarchy removes the need for this approximation, at the expense of requiring one for a higher-order moment of $f^{(1)}$.

Since these approximations are unconstrained, it is crucial to test them numerically. As far as we know, these are the first such verifications of a phase space 
DDFT. We now describe three such tests for hard spheres of diameter $\sigma$. We non-dimensionalize the equations with the units of length, mass and energy being $\sigma, m$ and $k_{B} T$ respectively. We set $f_{\text {neq }}^{(1)}=0$, use the hardsphere FMT functional [20, and choose $g$ to be the simplest possible (volume-exclusion) pair correlation function $g\left(\mathbf{r}_{1}, \mathbf{r}_{2},[\rho]\right)=1$ for $\left|\mathbf{r}_{1}-\mathbf{r}_{2}\right|>1$ and zero otherwise. Whilst not entirely consistent with the FMT approximation, this s sufficiently accurate for our purposes. For HI we choose the Rotne-Prager approximation 8, 22 in the overdamped limit and its inverse for $\boldsymbol{\Gamma}$ in (1). We use an 11-term two-body expansion [23] for $\boldsymbol{\Gamma}$ in (4), leading to small quantitative differences between (1) and (4). See Supplemental Material.

We take external potentials which depend spatially only on $\left|\mathbf{r}_{1}\right|$, and assume that the same holds for $\rho$ and $\mathbf{v}$, giving a 1D DDFT problem. We use spectral methods 24, appropriately extended to integral operators and a fifth order implicit Runge-Kutta method with step size control [25]. The infinite physical domain is mirrored and algebraically mapped onto $[-1,1]$ with 200 Chebyshev collocation points, avoiding the singularity at the origin. To capture the exponential decay of $\rho,(3)$ and (4) are reformulated for $\log \rho+V_{1}$. The initial condition is obtained from equilibrium DFT [19. We solve the stochastic equations via an Euler-Maruyama scheme with $10^{5}$ time steps, averaged over 5000 runs, with initial conditions chosen via slice sampling the (unnormalized) equilibrium $N$-body distribution. The hard sphere potential is approximated via a slightly softened, differentiable one 8 .

Fig. 11 shows the mean radial position and velocity of 50 particles, with $\gamma=6$, starting at equilibrium in a radially-symmetric external potential $V_{1}(r ; 3)$ with $V_{1}\left(r ; r_{0}\right)=0.1(1-h) r^{2}+3 h-10 \exp \left[-\left(r-r_{0}\right)^{2} / 4\right]$, where $h(r)=\left[\operatorname{erf}\left(\left(r+r_{0}\right) / 2\right)-\operatorname{erf}\left(\left(r-r_{0}\right) / 2\right)\right] / 2$ is a smooth cutoff. The potential is instantaneously switched to $V_{1}(r ; 0)$ at time 0 , and back to $V_{1}(r ; 3)$ at time 0.5 . The choice of 50 particles is large enough to overcome the differences between the canonical ensemble stochastic and grand canonical ensemble DDFT models, but also allows ease of access to stochastic simulations. We show four pairs of computations, each containing the solutions of a DDFT (lines) and the corresponding stochastic equation (symbols). The first pair (blue, solid) includes both inertia and HI and compares our DDFT (3) and (4) to the Euler-Maruyama [26] solution of (1) (circles). The second pair (red, long dashes, squares) are the same simulations, but when $\mathrm{HI}$ are neglected by setting $\boldsymbol{\Gamma}=\gamma \mathbf{1}$; see [9. The agreement between the DDFTs and stochastic simulations is very good. The HI effects are quite striking; they increase the effective friction and damp the dynamics.

The remaining two pairs of simulations in Fig. 1 are restricted to position space via the high-friction approximation. The DDFTs both with [8] (green, short dashes)

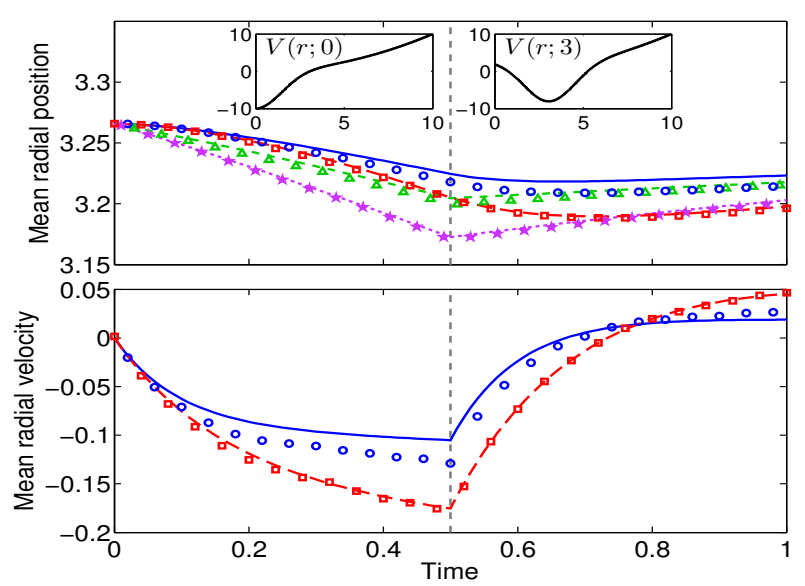

FIG. 1. Mean radial positions and velocities from DDFT (lines) and stochastic equations (symbols). Full phase space with (blue, solid, circles) and without (red, long dashes, squares) HI from DDFT (3) and (4) and stochastics (1). Overdamped limit DDFT [8] and stochastics [14] with (green, short dashes, triangles) and without (purple, dots, stars) HI.

and without [10] (purple, dots) HI, are compared to the Ermak-McCammon [14] solution of the corresponding stochastic equations (triangles, stars respectively). Whilst the agreement between DDFT and stochastic simulations is again very good, neglecting inertia leads to qualitatively different behaviour of the system, resulting in a kink in the mean position, compared to smooth curves with a delay before the mean velocity changes sign. Again, HI are seen to significantly damp the dynamics.

Fig. 2 shows the evolution of the same 50 particles, but we now switch between potentials $V_{1}(r ; 6)$ and $V_{1}(r ; 0)$ only once, at time 0 . We again have very good agreement between our DDFT and stochastic simulations. The small differences in the position distribution near the origin are likely due to the choice of correlation function, which is less accurate at higher densities. Here, HI dramatically slow the build-up of particles near the origin. Having verified our DDFT by comparison to stochastic simulations, Fig. 3 shows the DDFT solution for 500 particles with the same potentials, for which the stochastic equations are computationally very costly. The HI effects are even more dramatic, leading to qualitatively different behaviour. This size-dependence shows that HI must be carefully considered in any DDFT used to model macroscopic numbers of particles.

From now on we consider two-body inter-particle potentials and discuss two limits of (4). Close to local equilibrium, we expand $f^{(1)}$ and $f^{(2)}$ as Taylor series in $\boldsymbol{\nabla}_{\mathbf{r}_{1}} \mathbf{v}$ [6], obtaining a generalized compressible, non-local Navier-Stokes-like integro-differential equation:

$$
\rho D_{t} \mathbf{v}=\eta \boldsymbol{\nabla}_{\mathbf{r}_{1}}^{2} \mathbf{v}+\left(\zeta+\frac{1}{3} \eta\right) \boldsymbol{\nabla}_{\mathbf{r}_{1}}\left(\boldsymbol{\nabla}_{\mathbf{r}_{1}} \cdot \mathbf{v}\right)+\rho \mathcal{G}([\rho],[\mathbf{v}]),
$$

where $\mathbf{v}=\mathbf{v}\left(\mathbf{r}_{1}, t\right), \rho=\rho\left(\mathbf{r}_{1}, t\right)$ and $\mathcal{G}([\rho],[\mathbf{v}])$ is the right hand side of (4). The first three terms are standard but 

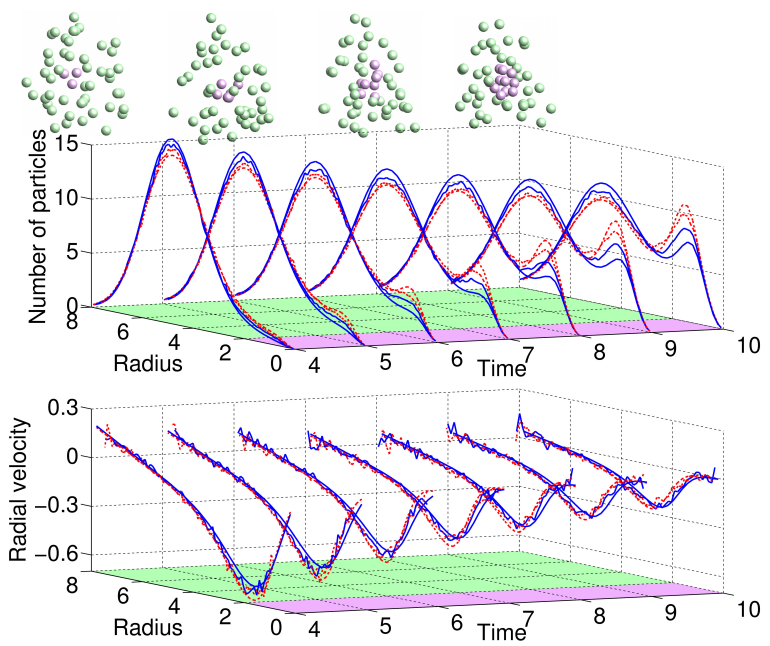

FIG. 2. Radial particle distribution and velocity from DDFT (3) and (4) (smooth curves) and stochastic equations (1) (noisy curves) with (blue, solid) and without (red, dashes) $\mathrm{HI}$. Also shown is one representative stochastic realization, at times 4, 6, 8 and 10, particles coloured purple for $|\mathbf{r}|<2$, green otherwise. See Supplemental Movies 2 and 3.
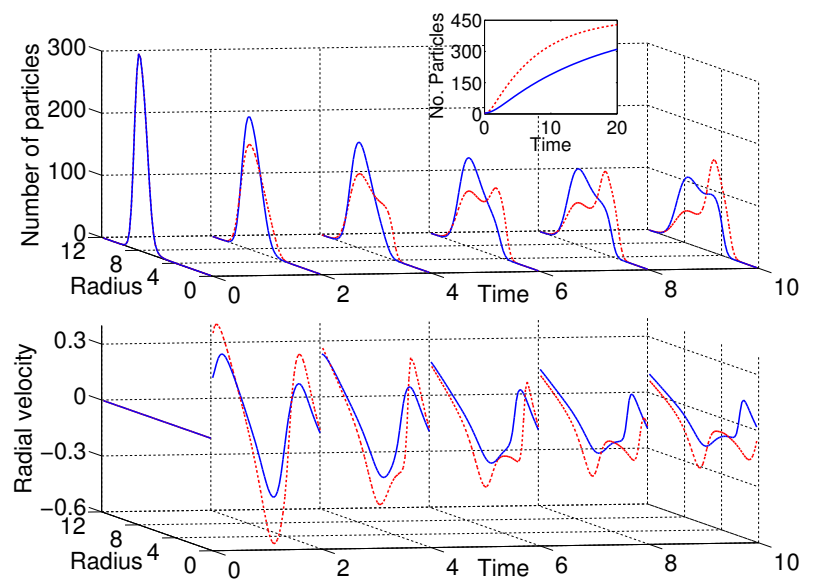

FIG. 3. Radial particle distribution and velocity from DDFT (3) and (4) with (blue, solid) and without (red, dashes) HI. Inset: number of particles with $0<|\mathbf{r}|<6$. See Supplemental Movie 4.

the viscosities $\eta$ and $\zeta$ are given by integrals involving the two-body potential and the Taylor expansion coefficients. Hence, the above equation is not amenable to a straightforward numerical solution, as also is the case for a simple fluid [6]. The new terms in $\mathcal{G}$ are a pressure-like term, depending on the gradient of the chemical potential, and HI terms, discussed above.

Most DDFTs are formulated in the high-friction regime, where the momenta of the colloidal particles equilibrate on a much shorter timescale than their positions. In this regime, we have a nondimensional parameter $\epsilon=\sqrt{k_{B} T / m} \gamma^{-1} L^{-1} \ll 1$, where $L$ is a 'typical' length scale of the system.
Denoting a Maxwellian momentum distribution by $M\left(\mathbf{p}_{1}\right)=\exp \left(-\left|\mathbf{p}_{1}\right|^{2} /\left(2 m k_{B} T\right)\right) /\left(2 m k_{B} T \pi\right)^{3 / 2}$, we find rigorously [11] that $f^{(1)}\left(\mathbf{r}_{1}, \mathbf{p}_{1}, t\right)=M\left(\mathbf{p}_{1}\right)\left(\rho\left(\mathbf{r}_{1}, t\right)+\right.$ $\left.\epsilon \mathbf{a}\left(\mathbf{r}_{1}, t\right) \cdot \mathbf{p}_{1}+\mathcal{O}\left(\epsilon^{2}\right)\right)$ for some function $\mathbf{a}$; in particular $\int \mathrm{d} \mathbf{p}_{1}\left(\mathbf{p}_{1} \otimes \mathbf{p}_{1}\right) f^{(1)}\left(\mathbf{r}_{1}, \mathbf{p}_{1}, t\right)=\mathcal{O}\left(\epsilon^{2}\right)$. For ease of presentation, we set $\mathbf{Z}_{2}=0$ (see [11] for the generalization to $\left.\mathbf{Z}_{2} \neq 0\right)$ and let $\rho^{(2)}\left(\mathbf{r}_{1}, \mathbf{r}_{2}, t\right)=\rho\left(\mathbf{r}_{1}, t\right) \rho\left(\mathbf{r}_{2}, t\right) g\left(\mathbf{r}_{1}, \mathbf{r}_{2}\right)$. Then $\rho$ satisfies a Smoluchowski equation [1] with a novel diffusion tensor

$\mathbf{D}\left(\mathbf{r}_{1},[\rho]\right)=\frac{k_{B} T}{m \gamma}\left[\mathbf{1}+\int \mathrm{d} \mathbf{r}_{2} g\left(\mathbf{r}_{1}, \mathbf{r}_{2}\right) \rho\left(\mathbf{r}_{2}, t\right) \mathbf{Z}_{1}\left(\mathbf{r}_{1}, \mathbf{r}_{2}\right)\right]^{-1}$,

retained in the DDFT, cf. [8]. Surprisingly, D is a nonlocal functional of $\rho$ and implicitly time-dependent, even though the friction tensor is time-independent. Previous phenomenological attempts at including a densitydependent diffusion coefficient in DDFTs do not correctly take into account the form of the diffusion tensor [27.

Our new DDFT should accurately model a wide spectrum of real-world problems and also help elucidate the associated underlying phenomena. These include systems in which HI or inertia are crucial, e.g. (i) wetting phenomena 28; (ii) transport and coagulation of nanoparticles in pulsatile and oscillatory systems [29]; and, (iii) cloud formation and deposition of nanoparticles [29]. Furthermore, there are many promising extensions to the modelling approach proposed here, e.g. to self-propelled particles, modelling bacteria; multiple particle species; anisotropic particles; and the inclusion of an external flow field, as would be required in modelling blood and drug-laden nanoparticle movement in blood. Similar approaches should also be highly relevant in granular media, ion transport, and other multi-phase systems.

We thank Petr Yatsyshin for stimulating discussions regarding free-energy functionals. We are grateful to the European Research Council via Advanced Grant No. 247031, the Rotary Clubs Darmstadt, DarmstadtBergstraße and Darmstadt-Kranichstein, the European Framework 7 via Grant No. 214919 (Multiflow) and the Engineering and Physical Sciences Research Council of the UK via grant No. EP/H034587/ for support of this research.

[1] R. Brown, Phil. Mag. 4, 161 (1828).

[2] A. Einstein, Ann. Phys. Lpz 17, 549 (1905); P. Langevin, C. R. Acad. Sci. (Paris) 146, 530 (1908); M. Von Smoluchowski, Ann. Phys 48, 1103 (1915).

[3] J. Perrin, Ann. Chim. Phys. 18, 1 (1909); T. Franosch, M. Grimm, M. Belushkin, F. Mor, G. Foffi, L. Forró, and S. Jeney, Nature 478, 85 (2011); R. Huang, I. Chavez, K. Taute, B. Lukić, S. Jeney, M. G. Raizen, and E. L. Florin, Nature Physics 7, 576 (2011).

[4] E. Matijević, ed., Medical Applications of Colloids (Springer, New York, 2008). 
[5] D. F. Evans and H. Wennerström, The Colloidal Domain: Where Physics, Chemistry, and Biology Meet (Wiley, New York, 1999); H. Stone and S. Kim, AIChE J. 47, 1250 (2001); F. Caruso, Colloids and Colloid Assemblies: Synthesis, Modification, Organization and Utilization of Colloid Particles (Wiley, Weinheim, 2004).

[6] H. J. Kreuzer, Nonequilibrium Thermodynamics and Its Statistical Foundations (Oxford University Press, New York, 1981).

[7] J. F. Lutsko, Adv. Chem. Phys. 144, 1 (2010).

[8] M. Rex and H. Löwen, Eur. Phys. J. E 28, 139 (2009).

[9] A. J. Archer, J. Chem. Phys. 130, 014509 (2009).

[10] U. M. B. Marconi and P. Tarazona, J. Chem. Phys. 110, 8032 (1999).

[11] B. D. Goddard, G. A. Pavliotis, and S. Kalliadasis, Multiscale Model. Sim. 10, 633 (2012).

[12] J. K. G. Dhont, An Introduction to Dynamics of Colloids (Elsevier, 1996).

[13] J. T. Padding and A. A. Louis, Phys. Rev. E 74, 031402 (2006).

[14] D. L. Ermak and J. A. McCammon, J. Chem. Phys. 69, 1351 (1978).

[15] G. K.-L. Chan and R. Finken, Phys. Rev. Lett. 94, 183001 (2005).

[16] M. A. L. Marques, C. A. Ullrich, F. Nogueira, A. Rubio, K. Burke, and E. K. U. Gross, eds., Time-Dependent Density Functional Theory, Lect. Notes Phys., Vol. 706 (Springer, Berlin, 2006).

[17] P. Résibois and M. De Leener, Classical Kinetic Theory of Fluids (Wiley, 1977).

[18] H. A. Knudsen, J. H. Werth, and D. E. Wolf, Eur. Phys. J. E 27, 161 (2008).
[19] R. Evans, Adv. Phys. 28, 143 (1979).

[20] Y. Rosenfeld, Phys. Rev. Lett. 63, 980 (1989).

[21] K. H. Hughes and I. Burghardt, J. Chem. Phys. 136, 214109 (2012).

[22] J. Rotne and S. Prager, J. Chem. Phys. 50, 4831 (1969).

[23] D. J. Jeffrey and Y. Onishi, J. Fluid Mech. 139, 261 (1984).

[24] J. P. Boyd, Chebyshev and Fourier Spectral Methods (Dover, UK, 2001).

[25] E. Hairer and G. Wanner, Solving Ordinary Differential Equations II. Stiff and Differential-Algebraic Problems, Springer Series in Computational Mathematics, Vol. 14 (Springer-Verlag, Berlin, 2006).

[26] P. E. Kloeden and E. Platen, Numerical Solution of Stochastic Differential Equations (Springer-Verlag, Berlin, 1992).

[27] M. Rauscher, J. Phys. Condens. Matter 22, 364109 (2010).

[28] P. Yatsyshin, N. Savva, and S. Kalliadasis, J. Chem. Phys. 136, 124113 (2012); A. Pereira and S. Kalliadasis, J. Fluid Mech. 692, 53 (2012); N. Savva, S. Kalliadasis, and G. Pavliotis, Phys. Rev. Lett. 104, 084501 (2010).

[29] P. Worth Longest and C. Kleinstreuer, J. Biomech. 36, 421 (2003); P. Worth Longest and J. Xi, J. Aerosol Sci. 38, 111 (2007); E. Gavze and M. Shapiro, J. Fluid Mech. 371, 59 (1998); H. R. Pruppacher, J. D. Klett, and P. K. Wang, Microphysics of clouds and precipitation (Taylor \& Francis, 1998); H. Sigurgeirsson and A. M. Stuart, Phys. Fluids 14, 4352 (2002); G. Falkovich, A. Fouxon, and M. Stepanov, Nature 419, 151 (2002). 